\title{
ASSISTÊNCIA AO BINÔMIO MÃE E LACTANTE COM COVID-19: UM RELATO DE EXPERIÊNCIA
}

\section{ASSISTANCE TO THE MOTHER AND BREASTFEEDING BINOMIUM WITH COVID-19: AN EXPERIENCE REPORT}

\author{
Maria Valéria Chaves de Lima ${ }^{1 *}$ Thaina Jacome Andrade de Lima ${ }^{2} *$ Kalyane Kelly Duarte de \\ Oliveira $^{3} *$ Vaniely Oliveira Ferreira $^{4} *$ Karina Morais Moura $^{5}$
}

\begin{abstract}
RESUMO
Objetivo: relatar a experiência de atendimento de uma equipe de Serviços de Atendimento Móvel de Urgência (SAMU) ao binômio mãe e lactente com Covid-19. Metodologia: trata-se de um relato de experiência. Resultados: o atendimento se deu em três momentos, executando-se uma triagem prévia e após uma anamnese detalhada foi constatado que no ato de amamentar a mãe não estava utilizando a máscara de proteção individual. A criança de 3 meses foi transportada para a unidade de atendimento mais próximo onde realizou teste para Covid-19, sendo positivo, contudo, não pode ser constatado a transmissão via leite materno, uma vez que as orientações de proteção e higiene não foram seguidas. Conclusão: fica clara a fundante necessidade de realizar a educação popular em saúde. Ressalta-se ainda a necessidade de atualização constante da equipe multiprofissional que presta atendimento de urgência e em especial aos pacientes com suspeita ou confirmados da Covid-19.
\end{abstract}

Palavras- Chaves: Aleitamento Materno; Infecção por corona vírus; Serviços Médicos de Emergência

\begin{abstract}
Objective: to report the experience of attending a Mobile Emergency Care Service (SAMU) team to the mother and infant binomial with Covid-19. Methodology: this is an experience report. Results: the service took place in three moments, performing a previous screening and after a detailed anamnesis it was found that in the act of breastfeeding the mother was not wearing the individual protection mask. The 3-month-old child was transported to the nearest care unit where he was tested for Covid-19, being positive, however, transmission via breast milk cannot be verified, since the protection and hygiene guidelines were not followed. Conclusion: the fundamental need for popular health education is clear. The need to constantly update the multiprofessional team that provides emergency care and especially to patients with suspected or confirmed patients at Covid-19 is also emphasized.
\end{abstract}

\footnotetext{
${ }^{1}$ Acadêmica de Enfermagem da Universidade do Estado do Rio Grande do Norte-UERN, Campus Avançado de Pau dos Ferros- CAPF. Pau dos Ferros, RN. Brasil.valerialima13@ hotmail.com, https://orcid.org/0000-0002-9278-5612

${ }^{2}$ Acadêmica de Enfermagem da Universidade do Estado do Rio Grande do Norte-UERN, Campus Avançado de Pau dos Ferros- CAPF Pau dos Ferros, RN. Brasil thainajacome@ hotmail.com https://orcid.org/0000-0003-1289-8842

${ }^{3}$ Doutora pelo Programa de Pós- Graduação em Enfermagem da Universidade Federal do Rio Grande do Norte- UFRN, Professora da Universidade do Estado do Rio Grande do Norte- UERN, Campus Avançado de Pau dos Ferros- CAPF. Mossoró, RN. Brasil kenfoliveira@gmail.com https://orcid.org/0000-0001-7713-3264

${ }^{4}$ Doutoranda Pela Universidade Estadual do Ceará-UECE. Professora da Universidade do Estado do Rio Grande do Norte-UERN, Campus Avançado de Pau dos Ferros- CAPF Pau dos Ferros, RN. Brasil vanielyvip@ hotmail.com https://orcid.org/0000-0002-3423-6885

${ }^{5}$ Acadêmica de Enfermagem da Universidade do Estado do Rio Grande do Norte-UERN, Campus Avançado de Pau dos Ferros- CAPF. Pau dos Ferros, RN. Brasil karinamoura17@ hotmail.com https://orcid.org/0000-0002-2402-4360
} 
Keywords: Breastfeeding; Infection from coronavirus; Emergency Medical Services

\section{INTRODUÇÃO}

A covid-19 é uma doença causada pelo vírus SARS-CoV-2, recentemente descoberto, pertencente à família dos corona vírus. Os primeiros sinais da doença surgiram no mês de dezembro de 2019 na província chinesa de Wuhan, mais especificamente em um mercado de frutos de mar. Pelo número considerável de contágios na China primeiramente declarouse surto da doença, logo após ela recebeu importância como emergência de saúde pública, e após chegar a 27 países e perpassa continentes, a Covid-19 alcançou o trágico patamar de pandemia (1).

De rápido contágio e com poucos estudos até então descobertos, em aproximadamente sete meses o vírus já é responsável por mais de 400 mil óbitos e 7 milhões de contaminados em todo o mundo. Diante disso, os sistemas de saúde precisaram se adaptar para prestar o atendimento adequado proporcionar cura e bem estar aos pacientes e acima de tudo diminuir o número de óbitos que cresce a cada dia (2).

No Brasil devido a modalidade descentralizada e hierarquizada do Sistema Único de Saúde (SUS) todos os níveis de atenção estão prestando serviços relacionados a Covid-19. Sendo que estes serviços estão organizando sua atenção desde a questão preventiva e de promoção à saúde, bem como nos âmbitos de assistência ambulatorial, de urgência e emergência. E isso tem sido facilitado por estruturas organizacionais como as redes de atenção à saúde (3).

O Serviço de Atendimento Móvel de Urgência (Samu) é um dos cinco componentes da Rede de Atenção à Saúde, e também da Política Nacional de Atenção às Urgências, que entrou em vigor no ano de 2003 com o objetivo de atender pessoas em situações de agravo de saúde e prestar um atendimento emergencial e de qualidade antes e durante o percurso dessa pessoa ao estabelecimento de saúde mais próximo. O acesso a esse serviço se dá através do telefone, discando o número 192(4). Conquanto, tendo em vista a emergência de saúde pública e sanitária atual, o SAMU passou a fazer os atendimentos e transportes de pacientes com Covid-19 em todo 


\section{RELATOS}

território brasileiro.

Ligada a estratégia de serviços em rede e pelo fato de que o Brasil, não alcançou a meta de redução da mortalidade materna e infantil, foram criadas algumas iniciativas para melhorar a qualidade da assistência às gestantes, puérperas e recém nascidos como a "Rede Cegonha" (2011) e a "Saúde Mais Perto de Você" (2013). Estas iniciativas têm, como foco, a melhoria nos quatro componentes: pré-natal; nascimento; puerpério e atenção integral à saúde da criança, além do sistema logístico, vinculado ao SAMU, que inclui os componentes transporte e regulação (5).

No contexto de pandemia destacamos a assistência ao puerpério, enfatizando o aleitamento materno. Os estudos preliminares discutem se há ou não transmissão da Covid-19 pelo leite materno, no entanto como o vírus é transmitido por gotículas, a forma como a amamentação acontece pode ser decisória para a contaminação ou não do lactente (6). Diante disso, a justificativa deste relato é a de proporcionar olhares sobre a amamentação na Covid-19. Entende-se que por esse ser um assunto novo, e com um grupo específico, será relevante para a prevenção, tratamento e redução da curva de contaminação da Covid19.

Assim objetivou-se relatar a experiência de atendimento de uma equipe de Serviços de Atendimento Móvel de Urgência (SAMU) ao binômio mãe e lactente com Covid-19.

\section{METODOLOGIA}

Trata-se de um estudo descritivo do tipo relato de experiência. Esta experiência surgiu de uma vivência em equipe, durante atendimentos do Serviço de Atendimento Móvel de Urgência (SAMU).

$\mathrm{O}$ relato deu-se após atendimento à ocorrência no Serviço de atendimento Móvel de Urgência (SAMU) Regional de Sousa/PB, o qual foi implantado na região no ano de 2005, respaldando-se no Decreto $\mathrm{n}^{\mathrm{a}}$ 5.055, de 27 de Abril de 2004 que institui o Serviço de Atendimento Móvel de Urgência-SAMU, em municípios e regiões do território nacional (7)

$\mathrm{O}$ pedido pelo atendimento do SAMU aconteceu no mês de maio, por uma família residente na Zona Rural do município.

Família composta de cinco pessoas, sendo uma criança com 3 meses em 


\section{RELATOS}

aleitamento materno exclusivo, a mãe, a adolescente e 2 adultos, que buscaram a rede de atendimento por alguns membros terem apresentados sintomatologia da Covid-19. Além da criança, dentro da estrutura familiar havia uma idosa que também se caracteriza grupos de risco para Covid-19. O atendimento se deu em três partes: triagem, detecção dos problemas e deslocamento para o serviço assistencial necessário.

A socialização da vivência é importante para que demais profissionais de saúde, acadêmicos possam se deparar com situações semelhantes. Ademais que ela pode servir como instrumento para mais estudos sobre a Covid-19 tendo em vista que é um tema novo e que requer mais pesquisas. Sendo desenvolvida através de uma vivência ocorrida após atendimento prestado pelo SAMU durante a pandemia da Covid-19, que exigiu dos profissionais entendimento sobre o assunto, agilidade e resolutividade.

$\mathrm{O}$ estudo não requer aprovação do comitê de ética, uma vez que as informações apresentadas são experiências pessoais vivenciadas. Contudo, ressalta-se que todos os princípios éticos foram seguidos conforme a resolução 466/2012.

\section{RESULTADOS E DISCUSSÕES}

Primeiramente realizou-se uma triagem para reconhecimento dos integrantes da família e quais os sintomas que cada um apresentava no momento. Verificou-se a temperatura dos componentes e começou-se a investigação quanto a sintomatologia, no momento nenhum integrante apresentava febre. Ao realizar-se uma anamnese prévia quanto a história pregressa observou-se que a mãe, e a criança lactente e a adolescente apresentaram sintomas da síndrome gripal nos dias anteriores à visita.

Em seguida tentou-se entender como estava acontecendo a nutrição da criança, tendo como alimento único e exclusivo o leite materno, sem problemas nesse processo, e com boa aceitação pela lactente ao leite.

Durante a anamnese descobriu-se que no ato de amamentar a mãe não estava utilizando a máscara de proteção individual alegando não ter sido orientada quanto ao manejo da criança nestas situações ou do risco de exposição da criança em um momento tão significativo, íntimo e conectivo entre mãe e bebê. Sabendo-se disso começou-se a orientação a mãe, sobre como continuar realizando o ato de maneira mais segura para o binômio mãe-bebê. 


\section{RELATOS}

Orientou-se a mãe quanto a busca de serviços de atenção psicossocial caso sentir se frágil pelas novas medidas durante o período de amamentação, a partir do momento que as restrições impostas pela pandemia atingem as questões de troca de olhares, identificação de rosto materno e toque, e proximidade entre mãe e filho que começam a construir-se no momento em que a amamentação acontece e que são importantes para o fortalecimento do vínculo.

Por fim a criança de 3 meses, a adolescente e a mãe foram transportados pelo SAMU para a unidade de atendimento mais próximo onde realizou teste para Covid-19. O teste das 3 teve resultado positivo. Os demais integrantes da família foram orientados a ficar em casa em isolamento até desaparecimento dos sintomas e testagem negativa deles. Orientou-se também o distanciamento da criança, da adolescente e da mãe, dos demais membros da família (pai e idosa) com o intuito de não transmitir a doença para os dois que não apresentaram sintomas, ainda que isso não descarte a possibilidade dos dois serem assintomáticos. Como desfecho do caso os sintomas dos 3 não se agravaram.

A experiência foi construtiva por permitir que a família se porte de modo mais seguro, ademais que, através dos conhecimentos discutidos e abordados a mãe não precisou suspender a amamentação, e a criança vai continuar sendo nutrida da melhor forma possível sem que o vínculo mãe e filho promovido através do aleitamento materno deixe de acontecer.

Ressalta-se também a importância dessa ação pelo fato de que a maioria das mulheres gestantes ou puérperas se encontram confusas quanto a maneira de se portar diante da positividade de covid-19 em um dos componentes do binômio mãe-filho. Tendo em vista as inúmeras controvérsias entre órgãos e estudos quanto a questão do aleitamento em tempos de pandemia, valoriza-se que as mães sejam orientadas quanto as formas mais seguras e possíveis para que o momento aconteça (8).

E diante dessa quantidade de duvidas bem como da necessidade de capacitar os profissionais, o próprio Ministério de Saúde Brasileiro, criou em maio dois documentos auto didáticos para gestantes e profissionais se orientarem quanto ao que deve ser feito nas situações de decisão de amamentação durante essa fase, na suspeita ou confirmação de covid na mãe ou no bebê e 


\section{RELATOS}

sobre a participação da família como fonte de apoio nesse momento. $\mathrm{O}$ primeiro documento é intitulado "Perguntas Frequentes - Amamentação e COVID-19" a obra é baseada em um documento maior produzido pela Organização Mundial de saúde chamado FAQ (Frequently Asked Questions - Breastfeeding and COVID-19 for health care workers). A versão brasileira conta com 20 perguntas que possuem respostas e subtópicos quanto a orientações. Mas de modo geral as indagações e respostas giram em torno de três temas gerais, sendo: as evidências já encontradas sobre covid no leite materno; os efeitos benéficos e protetivos do leite materno e do contato direto pele e a pele; e dos efeitos prejudiciais do uso de fórmulas e diferentes tipos de leite (9).

O segundo documento produzido pelo Ministério da Saúde chamado "Fluxo de Decisão para Amamentação no contexto da COVID-19" embora menor que o anterior merece destaque por além de apresentar um fluxograma quanto a medidas que devem ser tomadas com o binômio desde a maternidade apresenta também as questões subjetivas que envolvem a gestante/puérpera em relação a disponibilidade e aceitabilidade da amamentação antes ou após o tratamento, dando destaque a necessidade de maior apoio, acolhimento e escuta dos profissionais nesse momento ímpar (10).

Outros documentos importantes também destacam a preocupação quanto a resolutividade das dúvidas de mães e profissionais quanto a amamentação em meio a pandemia, a Sociedade Brasileira de Pediatria lançou também no mês de maio, a nota de alerta. "Aleitamento Materno em tempos de COVID-19 - recomendações na maternidade e após a alta". A nota faz um breve resumo sobre o panorama epidemiológico envolvendo puérperas e lactantes, estudos e pesquisas até então publicadas, e segue descrevendo as medidas mais cabíveis para sala de parto, alojamentos conjuntos, uti e dentro dos próprios lares. Embora seja um texto breve é um documento mais voltado para profissionais diferente do primeiro divulgado pelo ministério da saúde. (11)

Todos esses documentos são as evidências de como as puérperas e lactantes merecem atenção e possuem especificidades a serem analisadas nesse contexto. Pois, são seres que costumam estar inclusos em grupos de riscos pela quantidade de 


\section{RELATOS}

alterações fisiológicas que são sujeitos devido ao período de maternidade, e somado a maternidade surge-se a "maternagem" que é a busca pela proteção e segurança do bebê, o que os colocam como grupos de risco diante da pandemia do corona vírus (12).

Somado a isso, outros fatores também caracterizam o indivíduo como participante de um grupo de risco para a doença, sendo levados em conta os aspectos como a questão da imunidade, nutrição, prática ou não de exercícios físicos e as comorbidades pré-existentes. Algumas alterações são natas do próprio processo de envelhecimento natural, como alteração do sistema imunológico, as práticas de atividades físicas tornam-se cada vez mais difíceis de serem realizadas, assim como as doenças crônicas tornam-se cada vez mais presentes os idosos, sendo esse grupo mais propenso a possuírem uma predeterminação a sofrerem maiores impactos com síndromes respiratórias (13).

No caso das crianças, preocupa-se por essas ainda não estarem com os sistemas totalmente formados ou recuperados de um período de adaptações e com a imunidade fragilizada ou em formação (13).

No caso da pandemia da corona vírus além da atenção e cuidado direcionado aos grupos de risco tem se buscado bastante a identificação precoce dos sintomas da covid19, levantando-se o alerta a partir da sintomatologia referente a síndrome gripal aguda.

De acordo com o ministério da saúde síndrome gripal aguda são os casos cuja o paciente apresenta uma febre com início súbito ainda que referida, podendo ser acompanhada de tosse, garganta dolorida ou dificuldade respiratória, bem como outros sintomas, tais como: artralgia, mialgia e cefaleia. Atualmente devido ao contexto epidemiológico, toda síndrome gripal é tida como suspeita para Covid-19, por isso requer monitoramento e acompanhamento do suspeito para confirmação ou descarte da doença causada pelo Sars-Cov-2 (14).

O ministério da saúde traz que o leite materno é o alimento mais importante para as crianças por ser rico em nutrientes essenciais para seu crescimento saudável, além de que as crianças que recebem leite materno por tempo adequado apresentam menos chances de desenvolvimento de obesidade ou anemia e de doenças crônicas. $\mathrm{O}$ aleitamento materno exclusivo (AME) configura-se quando a criança só recebe leite 


\section{RELATOS}

maternolhumano até completar aproximadamente seis meses de vida, e é uma das práticas mais incentivadas desde o pré-natal da gestante (14).

Até então a maioria dos estudos discute-se mais predominantemente investigações quanto a transmissibilidade vertical da Covid-19 de mãe para filho, observou-se também questões de via de parto e troca de secreções. No entanto, em relação ao leite materno indica-se que este continue sendo oferecido a criança (se de acordo pela mãe, e se até então realizado com sucesso) desde que a mesma siga as recomendações de higienizar mãos e mamas, utilizar máscara de proteção, evitar falar durante o ato bem como tossir ou espirrar enquanto em contato com a criança, visando assim a diminuição das possibilidades de contágio. Uma medida também sugerida é por exemplo, a realização a retirada do leite, ou seja, a ordenha e o oferecimento do leite por uma pessoa que não apresentem sintomatologia ou comportamento de risco para contaminação por corona vírus. Vale lembrar que a retirada do leite deve seguir todo o passo a passo higiênico de ordenha, preconizado nas cartilhas do ministério da saúde (15).

\section{CONCLUSÕES}

Ao final do atendimento fica claro a fundante necessidade de realizar a educação popular em saúde tendo em vista a riqueza que se pode adquirir ao deixar que a população também exerça autonomia e opiniões quantos as atividades de promoção, prevenção, tratamento e recuperação de sua própria saúde.

Ressalta-se ainda a necessidade de atualização constante da equipe multiprofissional que presta atendimento de urgência e em especial aos pacientes com suspeita ou confirmados da Covid-19, por tratar-se de uma doença nova e estar em constante mudança. Tais atualizações, farão a diferença na prestação da assistência de qualidade e segura ao paciente, familiares e aos próprios profissionais de saúde.

Por fim, a experiência vivida torna-se um momento único de aprendizado tendo em visto o momento histórico pelo qual a humanidade está passando, bem como, para o crescimento profissional e pessoal de todos os membros da equipe multiprofissional do SAMU. Este relato contribuirá para profissionais, estudantes e pesquisadores se embasarem em situações semelhantes bem 


\section{RELATOS}

como serve para impulsionar estudos futuros sobre a temática.

As principais limitações do estudo estão relacionadas a poucas literaturas especificas sobre puérperas e lactantes para respaldar as ações realizadas de modo científico e as modificações constantes nas informações sobre a covid-19 à medida que os estudos avançam.

\section{REFERÊNCIAS:}

1. Dong E; Du H, Gardner L. Um painel interativo baseado na Web para rastrear a COVID-19 em tempo real. As doenças infecciosas da Lancet 2020 Fev.; 20 (5). 533-534. Disponível em: https://www.thelancet.com/journals/laninf/ar ticle/PIIS1473-3099(20)30120-1/fulltext.

Acesso em: 12 Jun. 2020.

2. Organização Pan-Americana da Saúde. Disponível em: https://www.paho.org/bra/?gclid=Cj0KCQj wgJv4BRCrARIsAB17JI6VoWEr_CMq2Q mMBQ3KoRwKkL9cj8fSzVkGDKXx654 WDwPc7vb0-xwaAj1OEALw_wcB Acesso em: 12 jun. 2020.

3. Vale EP, Rodrigues GM, Costa DP,
Queiroz JM, Lima DG, Medeiros LPF et al. Reorganização da Rede de Atenção à Saúde para o enfrentamento da COVID-19 no município de Canaã dos Carajás, Pará. APS em Revista, 2020 Jun.; 2 (2). 83-90.

Disponível em: https://apsemrevista.org/aps/article/view/101 156. Acesso em: 12 Jun. 2020.

\section{Ciconet RM, Marques GQ, Lima MADS.} Educação em serviço para profissionais de saúde do Serviço de Atendimento Móvel de Urgência (SAMU): relato da experiência de Porto Alegre-RS. Interface-Comunicação, Saúde, Educação, 2008 Jun./Set; 12 (26). 659-666, 2008. Disponível em: https://www.scielo.br/scielo.php?pid=S1414 $-32832008000300016 \&$ script=sci_arttext. Acesso em: 12 Jun. 2020

5. Objetivos de Desenvolvimento do Milênio (BR) [homepage na internet]. Os objetivos de desenvolvimento do milênio. Brasília: Governo Federal; 2017 [Acesso em 12 junho de 2020] disponível em: http://www.odmbrasil.gov.br/os-objetivosde-desenvolvimento-do-milenio.

6. Miranda VSG, Rech RS, Maahs MAP, 


\section{RELATOS}

Berbert MCB, Almeida ST. Fonoaudiologia, amamentação e COVID-19: informações aos fonoaudiólogos. CoDAS. Sociedade Brasileira de Fonoaudiologia, 2020 Mai; 32

$$
\text { 1-2. Disponível em: }
$$

https://www.scielo.br/scielo.php?pid=S2317

$-17822020000300201 \&$ script=sci_arttext.

Acesso em: 12 Jun. 2020.

7. Brasil. Decreto $\mathrm{n}^{\mathrm{a}} 5.055$, de 27 de abril de 2004. Institui o Serviço de Atendimento Móvel de Urgência - SAMU, em Municípios e regiões do território nacional, e dá outras providências. Diário Oficial da União. Brasília, 27 de abril de 2004. Disponível em: http://www.planalto.gov.br/ccivil_03/_ato20 04-2006/2004/decreto/d5055.htm_Acesso em: 12 Jun. 2020.

8. Martins-Filho PR, Santos VS, Santos Jr HP. To breastfeed or not to breastfeed? Lack of evidence on the presence of SARS-CoV-2 in breastmilk of pregnant women with COVID-19. Rev. Panam Salud Publica, Mai 2020; 44 (59). 1-5. Disponível em: https://scielosp.org/article/rpsp/2020.v44/e5 9/\#. Acesso em: 27 Jul. 2020.
9. Ministério da Saúde (BR). Secretária de Atenção Primária à Saúde. Perguntas Frequentes- Amamentação e Covid-19. Brasília (DF); 2020. Disponível em: https://portaldeboaspraticas.iff.fiocruz.br/bib lioteca/perguntas-frequentes-amamentacaoe-covid-19-ms/ Acesso em: 19 Ago. 2020.

10. Ministério da Saúde (BR). Secretaria de Atenção Primária a Saúde. Fluxo de Decisão para Amamentação no contexto da Covid-19. Brasília (DF); ago. 2020. Disponível em: https://portaldeboaspraticas.iff.fiocruz.br/bib lioteca/fluxo-de-decisao-para-amamentacaono-contexto-da-covid-19/ Acesso em: 19 Ago. 2020.

11. Sociedade Brasileira de Pediatria. Nota de Alerta. Aleitamento Materno em tempos de Covid-19 - Recomendações na maternidade e após a alta. Disponível em: https://www.sbp.com.br/fileadmin/user_ upload/22467f-NA_-

_AleitMat_tempos_Covid-19-

_na_matern_e_apos_alta.pdf Acesso em: 19 Ago. 2020.

12. Estrela FM, Silva KKS, Cruz MA, Gomes NP. Gestantes no contexto da 


\section{RELATOS}

pandemia. Physis: Revista de Saúde Coletiva,

materno exclusivo e alimentação 2020; 30 (2). e300215. Disponível em: complementar. Saúde em Redes, 2016; 2 (4). https://www.ims.uerj.br/wp383-392. Disponível em: content/uploads/2020/05/physis30_2_a15.pd http://revista.redeunida.org.br/ojs/index.php/ f. Acesso em: 27 Jul. 2020. rede-unida/article/view/794/pdf_52. Acesso em: 12 Jun. 2020.

13. Minussi BB, Paludo EA, Passos JPB, Santos MJ, Mocellin O, Maeyama MA. Recebido: 2020-07-29

Grupos de risco da COVID-19: a possível Aceito: 2020-09-02

relação entre o acometimento de adultos jovens "saudáveis" e a imunidade. Braz. J. Hea. Rev., 2020 Mar/Abr.; 3 (2). 3739-3762. Disponível em: http://www.brazilianjournals.com/index.php/ BJHR/article/view/9303/8445. Acesso em: 12 Jun. 2020.

14. Castro FA, Porto M. Protocolo institucional Manejo do paciente internado na enfermaria com COVID-19. 2nd pt. Disponível em: https://hcunicamp.com.br/wpcontent/uploads/2020/04/Protocoloenfermaria-COVID-19-Versao-2.pdf. Acesso em: 12 Jun. 2020.

15. Carvalho JLS, Cirino IP, Lima LHO, Sousa AF, Carvalho MF, Oliveira EAR. Conhecimento das mães sobre aleitamento 